\section{Influência da vitamina C na modulação autonômica cardíaca no repouso e durante o exercício isométrico em crianças obesas}

\section{The influence of vitamin $C$ on cardiac autonomic modulation at rest and during isometric exercise in obese children}

Fabio Alexandre dos Santos Lira 1

Maria do Socorro Brasileiro-Santos 2

Vanessa Vieira Lopes Borba 3

Maria José Carvalho Costa 4

Pricilla Regina Oliveira Fernandes Dantas 5

Amilton da Cruz Santos 6 Universidade Federal da Paraíba. João Pessoa, PB, Brasil. 2, 6 Departamento de Educação Física. Centro de Ciências da Saúde. Universidade Federal da Paraíba. Castelo Branco I. João Pessoa, PB, Brasil. CEP: 58.051-900. E-mail: amilton@pq.cnpq.br

\begin{abstract}
Objectives: to draw a comparison between obese and eutrophic children regarding cardiac autonomic modulation and to investigate the effect of vitamin $C$ supplements on cardiac autonomic modulation in obese children, at rest and during isometric exercise.

Methods: the study covered eight eutrophic $\left(I M C=18 \pm 1 \mathrm{Kg} / \mathrm{cm}^{2}\right)$ and 21 obese $(I M C=29 \pm$ $1 \mathrm{Kg} / \mathrm{cm}^{2}$ ) children aged between 8 and 11 years of both sexes, distributed into two groups: one receiving a $500 \mathrm{mg}$ vitamin $C$ supplement $(n=11)$, and the other a placebo $(n=10)$, for 45 days. Cardiac autonomic modulation was evaluated by way of spectral analysis of the variability of cardiac frequency at rest and during isometric exercise.

Results: obese children, at rest and during isometric exercise, presented a higher degree of sympathetic cardiac activation and less vagal activity. Comparison of the low- and high-frequency spectral components of the two groups revealed significant differences. After the use of vitamin C supplements, the spectral components were similar to those found in eutrophic children, both at rest and during isometric exercise.

Conclusions: obese children showed greater sympathetic activity and less vagal activity at rest and during isometric exercise compared to eutrophic children. The use of high doses of oral vitamin C supplements may correct the cardiac autonomic dysfunction in obese children.
\end{abstract}

Key words Obesity, Autonomic nervous system, Ascorbic acid, Heart hate

\section{Resumo}

Objetivos: comparar, entre obesos e eutróficos, os parâmetros de modulação autonômica cardíaca $e$ analisar o efeito da suplementação da vitamina $C$ na modulação autonômica cardíaca em crianças obesas, no repouso e durante o exercício isométrico respectivamente.

Métodos: participaram oito crianças eutróficas $\left(I M C=18 \pm 1 \mathrm{Kg} / \mathrm{cm}^{2}\right)$ e 21 obesas $(I M C=29 \pm$ $1 \mathrm{Kg} / \mathrm{cm}^{2}$ ), com idade entre 8 e 11 anos, ambos os gêneros, distribuidas em dois grupos: suplementadas com $500 \mathrm{mg}$ de vitamina $C(n=11)$, e placebo $(n=10)$, durante 45 dias. Modulação autonômica cardíaca foi avaliada por análise espectral da variabilidade da frequência cardíaca em repouso e durante o exercício isométrico.

Resultados: crianças obesas, no repouso e durante o exercício isométrico, apresentaram uma maior ativação simpática cardíaca e uma menor atividade vagal. Quando se comparou os componentes espectrais de baixa e alta frequência entre os grupos avaliados, observaram-se diferenças significativas. Após suplementação com vitamina $C$, os componentes espectrais foram similares àqueles observados nas crianças eutróficas, tanto no repouso como durante o exercício isométrico.

Conclusões: crianças obesas apresentam uma maior atividade simpática e menor atividade vagal no repouso e durante o exercício isométrico quando comparada as crianças eutróficas. Como também, a suplementação oral com vitamina $C$, em altas doses, pode restaurar a disfunção autonômica cardíaca em crianças obesas.

Palavras-chave Obesidade, Sistema nervoso autônomo, Vitamina C, Frequência cardíaca 


\section{Introdução}

A obesidade tem sido considerada um fator de risco independente para as doenças cardiovasculares, estando inclusive associada a uma maior morbidade e mortalidade. Evidência prévia demonstrou que a obesidade tem uma correlação positiva com os níveis de atividade simpática, que acarreta elevação dos níveis de pressão arterial e diminuição do fluxo sanguíneo muscular. ${ }^{1}$ Como resultado destas alterações, indivíduos obesos apresentam disfunção barorreflexa, ${ }^{2}$ resistência vascular periférica aumentada e estresse oxidativo elevado. ${ }^{3}$

Durante o exercício isométrico, vários reflexos são ativados conduzindo ao aumento da atividade nervosa simpática. Primeiramente, antes do início do exercício, a atividade neural central conhecida por "comando central" é ativada. ${ }^{4} \mathrm{O}$ comando central regula o aumento da atividade simpática constritora. Segundo, os "mecanorreceptores" quando estimulados pela contração muscular contribuem para aumentar o reflexo da atividade nervosa simpática. 5 Por fim, os metabólitos acumulados durante o exercício estimulam fibras nervosas aferentes quimiosensitivas chamadas "metaboreceptores musculares". Essas fibras, quando estimuladas, aumentam a atividade simpática para ambos os membros exercitados e não exercitados. Sabe-se ainda, que o metaboreflexo é o principal mecanismo reflexo que ativa o sistema nervoso simpático durante o exercício isométrico. 6 Estudo anterior7 demonstrou que durante a ativação do comando central/mecanorreceptores e ativação metabólica, obesos têm maior atividade nervosa muscular simpática, frequência cardíaca, pressão arterial comparada a normotensos eutróficos e que o controle do metaboreflexo da atividade nervosa simpática muscular está diminuído.

Adicionalmente, estresse oxidativo pode ser um problema importante na obesidade, uma vez que, níveis de antioxidantes reduzidos também podem alterar importantes funções fisiológicas, como o sistema nervoso autonômico, sensibilidade barorreflexa e bioatividade do óxido nítrico endotelial. Atualmente, substâncias antioxidantes têm sido recomendadas no tratamento do estresse oxidativo na obesidade 8 e em diferentes doenças cardiovasculares, 9,10 uma vez que, antioxidantes têm sido inversamente relacionados aos fatores de risco cardiovasculares, como obesidade, pressão sanguínea aumentada e concentração elevada de lipídeos circulantes. ${ }^{11,12}$ Elevadas doses de ácido ascórbico, podem agir reduzindo ânions superóxidos, enquanto que, sua terapia oral crônica pode aumentar a produção de óxido nítrico e/ou ativar a ação de antioxidantes. 9,13

O ácido ascórbico é conhecido principalmente por ser um antioxidante solúvel em água, que elimina ânions superóxidos e outras espécies reativas de oxigênio. Estudos realizados com obesos ${ }^{3}$ ou em outros grupos, como pacientes com insuficiência cardíaca 14 demonstraram que os níveis de ácido ascórbico estão diminuídos nestes quando comparado ao grupo controle. Para podermos utilizar uma terapia com antioxidante neste estudo, onde as crianças obesas podem estar expostas a uma condição de estresse oxidativo e hiperatividade simpática optou-se pela utilização da vitamina $C$, uma vez que, sua ação e segurança já estão demonstradas na literatura médica. 15-17

Notadamente, poucos estudos têm avaliado a resposta do sistema nervoso autonômico a manobras fisiológicas em crianças obesas suplementadas com vitamina C. Deste modo, o objetivo desta investigação foi comparar, entre obesos e eutróficos, os parâmetros de modulação autonômica cardíaca no repouso e durante o exercício isométrico, e analisar o efeito da suplementação da vitamina $\mathrm{C}$ na modulação autonômica cardíaca em repouso e durante o exercício isométrico em crianças obesas.

\section{Métodos}

As crianças obesas atenderam aos seguintes critérios de inclusão/exclusão: a) idade entre 8 a 11 anos; b) ambos os gêneros; c) índice de massa corporal (IMC) $>95 \%$; d) não estarem sob uso de medicamentos; e) não terem evidências de doenças cardiovasculares, renais, respiratórias, hormonais e/ou metabólicas durante a realização do estudo; e f) não estar envolvidas em programas de atividade física regular e sistematizadas. Elas foram divididas aleatoriamente em dois grupos, do tipo randomizado e duplo cego, as quais foram suplementadas com 500 $\mathrm{mg}$ de vitamina $\mathrm{C}(\mathrm{n}=11)$ ou substância placebo $(\mathrm{n}=10)$ durante 45 dias. A obesidade foi definida através do IMC específico para idade e gênero. Para comparar os valores da modulação autonômica cardíaca das crianças obesas com os das crianças eutróficas, oito crianças eutróficas, pareadas por idade e sexo foram engajadas no estudo (IMC entre 16 e $19 \mathrm{Kg} / \mathrm{m}^{2}$ ). Com exceção do IMC, todos os critérios de inclusão/exclusão utilizados para selecionar as crianças obesas foram utilizados para seleção das crianças eutróficas. Crianças eutróficas foram recrutadas entre aquelas que se matriculavam, pela primeira vez, nos programas de extensão do Departamento de Educação Física da Universidade 
Federal da Paraíba (UFPB), sem experiência prévia de atividade física regular e sistematizada. Este estudo foi aprovado pelo Comitê de Ética em Seres Humanos do Centro de Ciências da Saúde da Universidade Federal da Paraíba, protocolo $\mathrm{n}^{\mathrm{o}}$ 0464/2008.

Os experimentos foram realizados entre março e dezembro de 2009. Para triagem das crianças obesas foram consultados os prontuários de aproximadamente 200 crianças do Ambulatório de Endocrinologia do Hospital Universitário Lauro Wanderley da Universidade Federal da Paraíba (HULW-UFPB). Após observância dos critérios de inclusão-exclusão, aproximadamente um terço delas estavam aptas a serem incluídas no estudo. Posteriormente, com o convite para participação, 29 pais responderam nossa solicitação, entretanto, por dificuldades para atenderem plenamente as exigências do protocolo experimental tivemos uma perda amostral de oito participantes.

Após obtenção da assinatura no termo de consentimento pelos responsáveis legais, o peso corporal foi medido com escala eletrônica e as crianças estavam vestidas com roupas leves (short $\mathrm{e}$ camiseta). A estatura foi medida pelo estadiômetro do tipo Harpenden.

Amostras de sangue foram obtidas para determinação das concentrações séricas de colesterol total (método colesterol-oxidase, CHOP/PAP) e frações (HDL dosado por método Trinder Enzimático e a fração LDL foi calculada pela equação de Friedewald), triglicérides (Método Trinder Lipase/Oxidase/Peroxidase) e glicose (método glicose oxidase/ Peroxidase) através de ensaios colorimétricos enzimáticos (Arquitect c800, Abbott, Illinois, EUA).

A pressão arterial foi monitorada intermitentemente e de modo não invasivo através de um equipamento oscilométrico e automático (Dixtal ${ }^{\circledR}, \mathrm{DX}$ 2020; Manaus, Brasil). O manguito oclusor foi posicionado ao redor do tornozelo direito da criança e, em seguida, insuflado automaticamente a cada trinta segundos.

Frequência cardíaca foi monitorada continuamente, na derivação II do eletrocardiograma, e a frequência respiratória foi medida através da cinta respiratória, a qual contém sensores bilaterais que captam o sinal respiratório através da distensibilidade da caixa torácica. Os sinais foram obtidos com a ajuda de um conversor analógico/digital da WINDAQ/DATAQ DI200 - USA, com frequência de amostragem de $1000 \mathrm{~Hz}$ por canal.

O exercício isométrico foi realizado com o membro dominante utilizando um dinamômetro de preensão manual (Jamar ${ }^{\circledR}$, USA, modelo 5030J1). ${ }^{7}$ As crianças foram instruídas a manter a respiração normal durante a realização do exercício.

Os intervalos R-R e dos ciclos respiratórios foram identificados através da utilização de algoritmo implementado no WINDAQ Playback e, posteriormente, transformados em série temporal do intervalo RR (tacograma) e da respiração (respirograma). As leituras dos espectros de potência dos tacogramas e respirogramas foram realizadas pelo método autorregresivo na faixa de frequência de 0 a $0,4 \mathrm{~Hz}$. A densidade espectral de potência foi integrada em duas bandas de frequência de interesse, a alta frequência (AF) entre 0,15 e $0,4 \mathrm{~Hz}$ e baixa frequência (BF) entre 0,03 e $0,15 \mathrm{~Hz}$. Foi calculado também o balanço autonômico que é determinado pela razão entre as potências absolutas dos componentes de baixa e alta frequência. A análise espectral da respiração foi utilizada como controle, para avaliar as possíveis influências da frequência respiratória sobre o componente simpático, presente na banda de BF.

As crianças chegaram ao laboratório, 30 minutos antes do início do procedimento experimental. Antes de iniciar a instrumentação, foram orientadas a esvaziarem a bexiga e, em seguida, foram posicionadas em decúbito dorsal na maca. Posteriormente, foram colocados os eletrodos de superfície no tórax para captação do sinal eletrocardiográfico. Foi colocado no tornozelo direito o manguito oclusor para obtenção da pressão arterial e, para coleta do sinal respiratório foi colocado à cinta respiratória, circundando a caixa torácica com os sensores posicionados bilateralmente entre o quinto e oitavo espaço intercostal. Para realização do exercício isométrico, as crianças foram solicitadas a deixar os braços apoiado na maca ao lado do corpo e a informar a mão dominante, em sequência, o dinamômetro de preensão manual foi posicionado na mão dominante e realizado três tentativa de contração voluntária máxima. Posteriormente, foi calculada uma média das três tentativas.

A frequência cardíaca e a frequência respiratória foram registradas continuamente durante dez minutos em repouso. Em sequência, a pressão arterial, frequência cardíaca e respiratória foram registradas durante três minutos. O exercício isométrico foi realizado por três minutos a $30 \%$ da contração voluntária máxima. Os sinais digitalizados do eletrocardiograma e do ciclo respiratório foram registrados continuamente durante o exercício isométrico, enquanto que, os valores da pressão arterial foram coletados a cada 30 segundos.

A vitamina $\mathrm{C}$ e o placebo (dextrose) foram 
acondicionados em cápsulas e colocados em potes lacrados e codificados, sendo fabricada pela Farmácia Escola da Universidade Federal de Pernambuco. Baseado nos estudos de Deruelle e Baron 18 e Mc. Rae, ${ }^{19}$ os participantes do grupo experimental consumiram diariamente, durante 45 dias, $500 \mathrm{mg}$ de vitamina $\mathrm{C}$ e o grupo placebo recebeu dextrose.

Para testar a normalidade da distribuição dos dados foi utilizado o teste de Kolmogorov- Smirnov. As possíveis diferenças das características antropométricas, bioquímica sanguínea, frequência cardíaca, pressão arterial e modulação autonômica cardíaca nos grupos estudados, em repouso, antes e após suplementação de vitamina $\mathrm{C}$ ou substância placebo foram analisadas pelo teste $t$ Student para amostras dependentes e independentes conforme a conveniência. Os dados foram apresentados como média \pm erro-padrão da média. Foi aceito $p<0,05$ como nível de significância.

\section{Resultados}

As características antropométricas e pressóricas em crianças obesas e eutróficas no repouso estão apresentadas na Tabela 1. Pode-se observar que as variáveis idade e estatura são similares em crianças obesas e eutróficas $(p=0,38)$. Peso corporal e IMC foram estatisticamente diferentes. Quando se comparou as variáveis pressóricas elas foram sempre maiores no grupo de crianças obesas $(p=0,01)$.

As informações da pressão arterial média, frequência cardíaca e sua variabilidade estão apresentadas na Tabela 2. Pressão arterial média foi significativamente diferente entre os grupos estudados no repouso e durante o exercício isométrico. A comparação intragrupos mostrou que a pressão arterial média aumentou de forma significativa durante o exercício isométrico nos dois grupos avaliados. Frequência cardíaca também aumentou de forma significativa durante o exercício isométrico nos dois grupos. Entretanto, quando se fez a comparação entre os grupos, não foram observadas diferenças significativas nem no repouso nem durante o exercício isométrico. As unidades normalizadas de baixa frequência, alta frequência e o balanço autonômico cardíaco na condição repouso e durante o exercício isométrico foram estatisticamente diferentes quando se comparou os grupos estudados. Quando se fez a comparação dentro do grupo, verificou-se que os componentes normalizados de baixa e alta frequência, como também, o balanço autonômico aumentou significativamente durante o exercício isométrico.

Características antropométricas e metabólicas antes e depois da intervenção com vitamina $\mathrm{C}$ ou placebo estão apresentadas na Tabela 3. Idade, peso corporal, estatura, IMC e as variáveis metabólicas foram similares nas crianças obesas randomizadas para vitamina $\mathrm{C}$ ou placebo.

Intervenção com vitamina $\mathrm{C}$ ou placebo não causou mudanças significativas no colesterol total, colesterol HDL, colesterol LDL, triglicérides ou glicose nas crianças obesas no período estudado.

As medidas da frequência cardíaca e da sua variabilidade durante o exercício isométrico estão apresentas na Tabela 4. Antes da intervenção frequência cardíaca e os valores de baixa frequência, alta frequência e do balanço autonômico, não foram significativamente diferentes entre os grupos placebo e vitamina C. Entretanto frequência cardíaca e os valores de baixa frequência, alta frequência e balanço autonômico aumentaram de forma significativa durante o exercício isométrico nos dois grupos estudados quando comparado à condição repouso.

Quando observado as análises estatísticas no

Tabela 1

Características antropométricas e pressóricas em crianças obesas e eutróficas na condição repouso.

\begin{tabular}{|c|c|c|c|}
\hline Parâmetros & $\begin{array}{l}\text { Obesas } \\
(\mathrm{N}=21)\end{array}$ & $\begin{array}{l}\text { Eutróficas } \\
\qquad(\mathrm{N}=8)\end{array}$ & $p$ \\
\hline Idade (anos) & $10 \pm 0,4$ & $10 \pm 0,7$ & 0,80 \\
\hline Peso $(\mathrm{kg})$ & $64 \pm 4,0$ & $33 \pm 2,0$ & 0,01 \\
\hline Estatura (m) & $1,49 \pm 0,2$ & $1,48 \pm 0,1$ & 0,87 \\
\hline IMC (kg/cm²) & $29 \pm 1,0$ & $18 \pm 1,0$ & 0,01 \\
\hline PAS (mmHg) & $110 \pm 1,5$ & $101 \pm 0,2$ & 0,01 \\
\hline PAD $(\mathrm{mmHg})$ & $70 \pm 0,7$ & $65 \pm 0,8$ & 0,01 \\
\hline
\end{tabular}

$\mathrm{IMC}=$ índice de massa corporal; $\mathrm{PAS}=$ pressão arterial sistólica; $\mathrm{PAD}=$ pressão arterial diastólica. 
Valores da pressão arterial média, frequência cardíaca e sua modulação autonômica na condição repouso e durante o exercício isométrico em crianças obesas e eutróficas.

\begin{tabular}{|c|c|c|}
\hline Parâmetros & $\begin{array}{l}\text { Obesas } \\
(\mathrm{N}=21)\end{array}$ & $\begin{array}{l}\text { Eutróficas } \\
\qquad(\mathrm{N}=8)\end{array}$ \\
\hline \multicolumn{3}{|l|}{ PAM $(\mathrm{mmHg})$} \\
\hline Repouso & $83 \pm 1$ & $77 \pm 1^{+}$ \\
\hline Exercício isométrico & $95 \pm 3^{*}$ & $87 \pm 4^{* \dagger}$ \\
\hline \multicolumn{3}{|l|}{ FC (bpm) } \\
\hline Repouso & $83 \pm 2$ & $81 \pm 1$ \\
\hline Exercício isométrico & $98 \pm 2^{*}$ & $105 \pm 5^{*}$ \\
\hline \multicolumn{3}{|l|}{ BF (un) } \\
\hline Repouso & $71 \pm 3$ & $41 \pm 2^{+}$ \\
\hline Exercício isométrico & $81 \pm 4 *$ & $69 \pm 4^{* \dagger}$ \\
\hline \multicolumn{3}{|l|}{ AF (un) } \\
\hline Repouso & $29 \pm 3$ & $59 \pm 2^{+}$ \\
\hline Exercício isométrico & $19 \pm 4^{*}$ & $31 \pm 3^{*+}$ \\
\hline \multicolumn{3}{|l|}{$\mathrm{BF} / \mathrm{AF}$} \\
\hline Repouso & $2,5 \pm 0,3$ & $0,7 \pm 0,1^{\dagger}$ \\
\hline Exercício isométrico & $4,3 \pm 0,4^{*}$ & $2,2 \pm 0,3^{*+}$ \\
\hline
\end{tabular}

PAM = pressão arterial média; $F C=$ frequência cardíaca; $B F($ un) = baixas frequências em unidades normalizadas; $\mathrm{AF}(\mathrm{un})=$ altas frequências em unidades normalizadas; $\mathrm{BF} / \mathrm{AF}=$ balanço autonômico; * $p<0,05$ repouso vs exercício isométrico; ${ }^{\dagger} p<0,05$ comparação entre grupos.

Tabela 3

Influência das intervenções nas características antropométricas e metabólicas em crianças obesas.

\begin{tabular}{|c|c|c|c|c|}
\hline \multirow[t]{2}{*}{ Parâmetros } & \multicolumn{2}{|c|}{$\begin{array}{c}\text { Vitamina C } \\
\qquad(\mathrm{N}=11)\end{array}$} & \multicolumn{2}{|c|}{$\begin{array}{r}\text { Placebo } \\
(\mathrm{N}=10)\end{array}$} \\
\hline & Antes & Depois & Antes & Depois \\
\hline Peso $(\mathrm{kg})$ & $64 \pm 2$ & $60 \pm 5$ & $64 \pm 2$ & $62 \pm 3$ \\
\hline Altura (m) & $1,48 \pm 0,2$ & $1,50 \pm 0,1$ & $1,49 \pm 0,2$ & $1,50 \pm 0,1$ \\
\hline $\mathrm{IMC}\left(\mathrm{kg} / \mathrm{cm}^{2}\right)$ & $29 \pm 5$ & $26 \pm 2$ & $28 \pm 2$ & $27 \pm 1$ \\
\hline Colesterol Total (mg/DI) & $160 \pm 17$ & $158 \pm 10$ & $167 \pm 15$ & $167 \pm 5$ \\
\hline Colesterol HDL (mg/DI) & $44 \pm 5$ & $44 \pm 1$ & $49 \pm 11$ & $49 \pm 10$ \\
\hline Colesterol LDL (mg/DI) & $91 \pm 10$ & $89 \pm 12$ & $90 \pm 10$ & $90 \pm 10$ \\
\hline Triglicérides (mg/DI) & $110 \pm 18$ & $105 \pm 9$ & $99 \pm 14$ & $100 \pm 7$ \\
\hline Glicose (mg/Dl) & $80 \pm 8$ & $82 \pm 10$ & $90 \pm 3$ & $89 \pm 5$ \\
\hline
\end{tabular}

IMC= índice de massa corporal; HDL= lipoproteína de alta densidade; LDL= lipoproteína de baixa densidade.

grupo de crianças obesas, antes versus depois da intervenção, vitamina $\mathrm{C}$ e placebo não alteraram de forma significativa os valores de frequência cardíaca no repouso ou durante o exercício isométrico. Por outro lado, quando comparado os valores de baixa frequência, alta frequência e o balanço autonômico cardíaco observou-se que eles foram significativamente diferentes no grupo que recebeu vitamina $\mathrm{C}$, no repouso e durante o exercício isométrico.

Adicionalmente, quando comparado os dois grupos estudados depois da intervenção, observouse que frequência cardíaca não foi estatisticamente 
diferente no repouso nem durante o exercício isométrico. Baixa frequência, alta frequência e balanço autonômico cardíaco foram significativamente diferentes entre os dois grupos estudados, tanto no repouso como durante o exercício isométrico (Tabela 4).

Por fim, fizemos a comparação da modulação autonômica cardíaca entre o grupo de crianças obesas pós-suplementação com vitamina $\mathrm{C}$ e o grupo de crianças eutróficas. Nesta avaliação, a vitamina C restaurou a modulação autonômica cardíaca nas crianças obesas, no repouso e durante o exercício isométrico, quando comparada com as crianças eutróficas $\left[\mathrm{BF}_{\text {repouso }}(45 \pm 5\right.$ vs $41 \pm 2$ un, $p=0,44)$, $\mathrm{BF}_{\text {exercício }}(57 \pm 5$ vs $63 \pm 4$ un, $p=0,25) ; \mathrm{AF}_{\text {repouso }}$ ( $55 \pm 5$ vs $59 \pm 2$ un, $p=0,44), \mathrm{AF}_{\text {exercício }}(43 \pm 5 v s$ $37 \pm 3$ un, $p=0,22) ; \mathrm{BF} / \mathrm{AF}_{\text {repouso }}(0,8 \pm 0,2$ vs $0,7 \pm$ $0,2, p=0,22)$ e $\mathrm{BF} / \mathrm{AF}_{\text {exercício }}(1,3 \pm 0,1$ vs $1,7 \pm 0,3$ $p=0,69)$ ] (Tabelas 2 e 4 ).

Tabela 4

Influência das intervenções na frequência cardíaca e sua modulação autonômica na condição repouso e durante o exercício isométrico em crianças obesas.

\begin{tabular}{|c|c|c|c|c|}
\hline \multirow[t]{2}{*}{ Parâmetros } & \multicolumn{2}{|c|}{$\begin{array}{c}\text { Vitamina C } \\
(\mathrm{N}=11)\end{array}$} & \multicolumn{2}{|c|}{$\begin{array}{r}\text { Placebo } \\
(N=10)\end{array}$} \\
\hline & Antes & Depois & Antes & Depois \\
\hline \multicolumn{5}{|l|}{$\mathrm{FC}(\mathrm{bpm})$} \\
\hline Repouso & $83 \pm 3$ & $86 \pm 3$ & $82 \pm 3$ & $82 \pm 3$ \\
\hline Exercício isométrico & $100 \pm 5^{*}$ & $103 \pm 5^{*}$ & $96 \pm 2 *$ & $95 \pm 2^{*}$ \\
\hline \multicolumn{5}{|l|}{ BF (un) } \\
\hline Repouso & $71 \pm 4$ & $45 \pm 5^{+}$ & $71 \pm 3$ & $65 \pm 2 \ddagger$ \\
\hline Exercício isométrico & $81 \pm 4^{*}$ & $57 \pm 5^{+}$ & $79 \pm 1$ * & $81 \pm 2^{* \neq}$ \\
\hline \multicolumn{5}{|l|}{$A F(u n)$} \\
\hline Repouso & $29 \pm 3$ & $55 \pm 5^{\dagger}$ & $29 \pm 3$ & $35 \pm 2 \ddagger$ \\
\hline Exercício isométrico & $19 \pm 4 *$ & $43 \pm 5^{\dagger}$ & $21 \pm 2 *$ & $19 \pm 2 * \neq$ \\
\hline \multicolumn{5}{|l|}{$\mathrm{BF} / \mathrm{AF}$} \\
\hline Repouso & $2,5 \pm 0,3$ & $0,8 \pm 0,2^{\dagger}$ & $2,5 \pm 0,3$ & $2,0 \pm 0,3 \ddagger$ \\
\hline Exercício isométrico & $4,3 \pm 0,4 *$ & $1,3 \pm 0,1 *+$ & $3,8 \pm 0,2^{*}$ & $4,3 \pm 0,4^{* \neq}$ \\
\hline
\end{tabular}

$\mathrm{FC}=$ frequência cardíaca; $\mathrm{BF}$ (un) = baixas frequências em unidades normalizadas; $\mathrm{AF}$ (un) = altas frequências em unidades normalizadas; $\mathrm{BF} / \mathrm{AF}=$ balanço autonômico; ${ }^{*} p<0,05$ repouso vs exercício isométrico; ${ }^{\dagger} p<0,05 \mathrm{Antes} v \mathrm{~s}$ Depois; $¥ p<0,05$ vitamina $C$ depois da intervenção vs placebo depois da intervenção.

\section{Discussão}

Este estudo demonstrou que em crianças obesas: a) ocorreu uma maior ativação simpática cardíaca e uma menor resposta vagal durante o exercício isométrico quando comparada às crianças eutróficas; b) a modulação autonômica cardíaca foi alterada significativamente após suplementação com vitamina $\mathrm{C}$ quando comparada ao grupo placebo; e, c) vitamina $\mathrm{C}$ restaurou a modulação autonômica cardíaca no repouso e durante o exercício isométrico quando comparada às crianças eutróficas.

As oscilações nas potências das bandas de baixa frequência e alta frequência caracterizam a variabilidade de frequência cardíaca. As mudanças no balanço autonômico ou simpatovagal ocorrem durante numerosas condições fisiológicas e fisiopatológicas, inclusive na obesidade infantil.20,21 Neste estudo, foram encontrados maiores valores de modulação simpática cardíaca no grupo de crianças obesas na condição repouso e durante o exercício isométrico. Desta forma, se esperava encontrar valores de pressão arterial e frequência cardíaca maiores nas crianças obesas no repouso e durante o exercício isométrico, contudo, isso não foi observado. Pressão arterial média foi maior nas crianças obesas no repouso e durante o exercício isométrico, corroborando com os achados da literatura.22,23 Contudo, os valores da frequência cardíaca não foram significativamente diferentes daqueles encon- 
trados nas crianças eutróficas no repouso ou durante o exercício isométrico.

Estudos têm demonstrado que o aumento da modulação simpática está associado à redução do controle da frequência cardíaca via parassimpático, uma vez que, os níveis de norepinefrina plasmática elevados em obesos modificam os valores das baixas frequências, 24 e isso pode reduzir a responsividade adrenoreceptora, 25 aumentar a atividade simpática 26 e diminuir a sensibilidade barorreflexa. 27 Informações adicionais sobre o papel do barorreflexo no desenvolvimento da hipertensão primária em crianças e adolescentes têm trazido subsídios preocupantes. Em crianças obesas, a sensibilidade barorreflexa se modifica para compensar a influência da atividade simpática na pressão sanguínea e a pressão arterial média permanece normal, e um novo set point para o barorreflexo é alcançado. Com a manutenção da atividade simpática e do estresse oxidativo elevados, o aumento da pressão sanguínea não é suficiente para suprir o barorreflexo, sendo assim, um novo set point em valores mais altos de pressão sanguínea é alcançado e a hipertensão é uma consequência. 28

Em sequência, foi testado à hipótese de que vitamina $C$, quando administrada cronicamente em elevadas doses, poderia restaurar a modulação autonômica cardíaca, em repouso e durante o exercício isométrico, quando comparado ao grupo placebo. Quando se verificou os valores do componente normalizado de baixa frequência e o balanço autonômico, que são indicadores da atividade simpática, estes continuavam aumentados no grupo de crianças que receberam placebo. Em crianças obesas, a ativação simpática tem sido considerada responsável pelo aumento da resistência vascular periférica muscular e, em consequência da pressão arterial, 24 que são fortes preditores de morbidade e mortalidade cardiovascular.

Vitamina C é conhecida especialmente por ser um antioxidante que elimina ânions superóxidos e outras espécies reativas de oxigênio, por isso, acreditamos que em nosso estudo, o estresse oxidativo pode ter sido reduzido no grupo de crianças obesas que receberam vitamina $\mathrm{C}$. $\mathrm{O}$ fato da vitamina $\mathrm{C}$ ser um antioxidante capaz de restaurar a modulação autonômica cardíaca não é recente, estudos prévios têm demonstrado que infusão intravenosa de vitamina $\mathrm{C}$ restaura a resposta da modulação autonômica cardíaca na insuficiência cardíaca. ${ }^{29,30}$ Esses autores têm demonstrado que um dos mecanismos prováveis para explicar a ação da vitamina $\mathrm{C}$ é que ela está atuando na inativação dos radicais livres, resultantes do estresse oxidativo elevado. Deste modo, no nosso estudo, podemos, em parte, atribuir às mudanças positivas na modulação autonômica cardíaca à inativação dos radicais livres pela administração da vitamina $C$, já que não houve alterações significativas no peso corporal, IMC ou perfil metabólico, fatores que poderiam também, estar envolvidos nessa melhoria. Por fim, podemos sugerir com os nossos resultados que, provavelmente, o estresse oxidativo gerou alterações na modulação autonômica cardíaca nas crianças obesas, já que a sua resposta foi diminuída pós-suplementação com vitamina $\mathrm{C}$.

Neste estudo, a resposta da modulação simpática cardíaca foi alterada após suplementação com vitamina $\mathrm{C}$, tanto no repouso como durante o exercício isométrico, entendemos que essas alterações foram importantes, uma vez que, os valores das potências nas bandas de baixa frequência e alta frequência normalizada, como também, do balanço autonômico se aproximam daqueles observados nas crianças eutróficas. Um dos prováveis mecanismos para explicar essa redução da atividade simpática, pode ser a melhoria da sensibilidade barorreflexa, via diminuição do estresse oxidativo. Estudos têm demostrado que na insuficiência cardíaca, por exemplo, a redução da sensibilidade barorreflexa ocorre principalmente pelo aumento da atividade simpática e, que vitamina $\mathrm{C}$ foi capaz de restaurar a sensibilidade barorreflexa nestes pacientes.29,31 É importante ressaltar que vitamina $\mathrm{C}$ tem sido reconhecida como um potente agente antioxidante e esse efeito tem sido sugerido, principalmente, nos estudos randomizados e transversais. 15,16 O EPIC-Norfolk prospective study: a prospective population study 17 observou uma forte relação entre a utilização da vitamina $\mathrm{C}$ e a redução de eventos coronarianos, também reconheceu, que a ingestão de vitamina $\mathrm{C}$ que excede $50 \mathrm{mg} /$ dia diminui a mortalidade para todas as doenças cardiovasculares.

Em conclusão, os resultados observados neste estudo demonstraram que suplementação oral com $500 \mathrm{mg}$ de vitamina $\mathrm{C}$ por 45 dias pode restaurar a modulação autonômica cardíaca em crianças obesas na condição repouso e durante o exercício isométrico a valores próximo da normalidade. Por fim, como não podemos assegurar o (s) mecanismo (s) envolvido neste restabelecimento, sugerimos que estudos prospectivos utilizando outros marcadores e/ou recursos metodológicos possam ser realizados com um número maior de participantes. Não foi possível dosar a LDL oxidada, um indicador do estresse oxidativo. A atividade autonômica cardíaca foi avaliada apenas no domínio da frequência. 


\section{Agradecimentos}

Os autores agradecem a direção da Farmácia Escola da Universidade Federal de Pernambuco pela

\section{Referências}

1. Ribeiro MM, Trombetta IC, Batalha LT, Rondon MUPB, Forjaz CLM, Villares SMF, Negrão CE. Muscle sympathetic nerve activity and hemodynamic alterations in middle-aged obese women. Braz J Med Biol Res. 2001; 34: 475-8.

2. Grassi G, Seravalle G, Colombo M, Bolla G, Cattaneo BM, Cavagnini F, Mancia G. Body weight reduction, sympathetic nerve traffic, and arterial baroreflex in obese normotensive humans. Circulation. 1998; 97: 2037-42.

3. Perticone F, Ceravolo R, Candigliota M, Ventura G, Iacopino S, Sinopoli F, Mattioli PL. Obesity and body fat distribution induce endothelial dysfunction by oxidative stress: protective effect of vitamin C. Diabetes. 2001; 50: 159-65.

4. Bevegard BS, Shepherd JT. Regulation of the circulation during exercise in man. Physiol Rev. 1967; 47: 178-213.

5. Vissing SF, Scherrer U, Victor RG. Stimulation of skin sympathetic nerve discharge by central command: differential control of sympathetic outflow to skin and skeletal muscle during static exercise. Circ Res. 1991; 69: 228-38.

6. Ray CA, Mark AL. Sympathetic nerve activity to nonactive muscle of the exercising and nonexercising limb. Med Sci Sports Exerc. 1995; 27: 183-7.

7. Negrão CE, Trombetta IC, Batalha LT, Ribeiro MM, Rondon MUPB, Tinucci T, Forjaz CLM, Barretto ACP, Halpern A, Villares SM F. Muscle metaboreflex control is diminished in normotensive obese women. Am J Physiol Heart Circ Physiol. 2001; 281: H469-75.

8. Carr AC, Frei B. Toward a new recommended dietary allowance for vitamin $\mathrm{C}$ basead on antioxidant and health effects in humans. Am J Clin Nutr. 1999; 69: 1086 -107.

9. Ellis GR, Anderson RA, Lang D, Blackman DJ, Morris RHK, Morris-Thurgood J, McDowell IFW, Jackson SK, Lewis MJ, Frenneaux MP. Neutrophil superoxide aniongenerating capacity, endothelial function and oxidative stress in chronic heart failure: effects of short- and longterm vitamin C therapy. J Am Coll Cardiol. 2000; 36: 147482.

10. Taddei S, Virdis A, Ghiadoni L, Magagna A, Salvetti A. Vitamin $\mathrm{C}$ improves endothelium-dependent vasodilation by restoring nitric oxide activity in essential hypertension. Circulation. 1998; 97: 2222-9.

11. Ness AR, Khaw KT, Bingham S, Day NE. Vitamin C and blood pressure. J Hypertens. 1996; 14: 503-8.

12. Ness AR, Khaw KT, Bingham S, Day NE. Vitamin C and serum lipids. Eur J Clin Nutr. 1996; 50: 724-9.

13. Yücel D, Aydogdu S, Cehreli S, Saydam G, Canatan H, Seneş M, Cigdem Topkaya B, Nebioglu S. Increased oxidative stress in dilated cardiomyopathic heart failure. Clin Chem. 1998; 44: 148-54.

14. Hornig B, Arakawa N, Kohler C, Drexler H. Vitamin C produção e doação da vitamina C e substância placebo utilizado neste estudo.

improves endothelial function of conduit arteries in patients with chronic heart failure. Circulation. 1998; 97: 363-8.

15. MRC/BHF. (Heart protection study collaborative group). Heart protection study of antioxidant vitamin supplementation in 20536 high-risk individuals: a randomized placebocontrolled trial. Lancet. 2002; 360: 23-33.

16. Enstrom JE. Vitamin C intake and mortality among sample of U.S. population. Epidemiol. 1992; 3: 194-202.

17. Khaw KT, Bingham S, Welch A, Luben R, Wareham N, Oakes S, Day N. Relation between plasma ascorbic acid and mortality in men and women in EPIC-Norfolk prospective study: a prospective population study. Lancet. 2001; 357: 657-63.

18. Deruelle F, Baron B. Vitamin C: is supplementation necessary for optimal health? J Altern Complement Med. 2008; 14: $1291-8$.

19. McRae MPI. Vitamin C effective antihypertensive supplement? A review and analysis of the literature. J Chiropr Med. 2006; 5: 60-4.

20. Rodríguez-Colón SM, Bixler EO, Li X, Vgontzas AN, Liao D. Obesity is associated with impaired cardiac autonomic modulation in children. Int J Pediatr Obes. 2011; 6: 128-34.

21. Tascilar ME, Yokusoglu M, Boyraz M, Baysan O, Köz C, Dündaröz R. Cardiac autonomic functions in obese children. J Clin Res Pediatr Endocrinol. 2011; 3: 60-4.

22. Ribeiro MM, Silva AG, Santos NS, Guazzelle I, Matos LNJ, Trombetta IC, Halpern A, Negrão CE, Villares SMF. Diet and exercise training restore blood pressure and responses during maneuvers in obese children. Circulation. 2005; 111: 1915-23.

23. Dantas PROF, Lira FAS, Borba VVL, Costa MJC, Brasileiro-Santos MS, Santos AC. Vitamin C restores blood pressure and vasodilator response during mental stress in obese children. Arq Bras Cardiol. 2011: 96; 490-7.

24. Zhou Y, Xie G, Wang J, Yang S. Cardiovascular risk factors significantly correlate with autonomic nervous system activity in children. Can J Cardiol. 2012; 28: 477-82.

25. Jung RT, Shetty PS, James WP, Barrand MA, Callingham BA. Plasma catecholamines and autonomic responsiveness in obesity. Int J Obes Res Metab Disord. 1982; 6: 131-41.

26. Gustafson AB, Kalkhoff RK. Influence of sex and obesity on plasma catecholamine response to isometric exercise. $\mathrm{J}$ Clin Endocr Metab. 1982; 55: 703-8.

27. Skrapari I, Tentolouris N, Katsilambros N. Baroreflex function: determinants in healthy subjects and disturbances in diabetes, obesity and metabolic syndrome. Curr Diabetes Rev. 2006; 2: 329-38.

28. Honzíková N, Nováková Z, Závodná E, Paděrová J, Lokaj P, Fišer B, Balcárková P,Hrstková H: Baroreflex sensitivity in children, adolescents, and young adults with essential and white-coat hypertension. Klin Paed. 2006; 218: 237-42. 
29. Piccirillo G, Nocco M, Moisè A, Lionetti M, Naso C, di Carlo S, Marigliano V. Influence of vitamin C on baroreflex sensitivity in chronic heart failure. Hypertension. 2003; 41: 1240-5.

30. Piccirillo G, Raffaele Q, Fimognari F, Moisè A, Mario M, Lionetti M, Naso C, Di Carlo S, Nocco M, Magrì D. Influence of L-arginine and vitamin $\mathrm{C}$ on the autonomic nervous system in chronic heart failure secondary to ischemic cardiomyopathy. Am J Cardiol. 2004; 93: 650-4.
31. Nightingale AK, Blackman DJ, Field R, Glover NJ, Pegge N, Mumford C, Schmitt M, Ellis GR, Morris-Thurgood JA, Frenneaux MP. Role of nitric oxide and oxidative stress in baroreceptor dysfunction in patients with chronic heart failure. Clin Sci. 2003; 104: 529-35.

Recebido em 25 de abril de 2011

Versão final apresentada em 28 de maio de 2012

Aprovado em 11 de junho de 2012 